\title{
MALFORMACIÓN CONGÉNITA DE LA VÍA AÉREA PULMONAR
}

\author{
Juan Carlos Tasayco Saravia ${ }^{1 a}$, Claudia Saldaña Diaz ${ }^{1 b}$, Juan Carlos Roque ${ }^{2 b}$, Jorge Segundo Paredes ${ }^{1 b}$, \\ Marcos Espinola Sánchez ${ }^{1 \mathrm{~b}}$.
}

\begin{abstract}
RESUMEN
La malformación congénita de la vía aérea pulmonar (MCVAP), antiguamente conocida como malformación adenomatoide quística congénita del pulmon (MAQP), pertenece al grupo de las malformaciones congénitas pulmonares, representando al $95 \%$ de estas. Se caracteriza por distintos grados de distress respiratorio según el nivel de la vía aérea que comprometa la lesión pudiendo ser desde incompatible con la vida hasta pasar inadvertida hasta adultez temprana. Los adelantos de la biología molecular han descubierto que existe un disbalance entre los genes proliferadores y apoptoticos dentro de estas lesiones interrumpiendo la segmentación del árbol bronquial desencadenando la formación de estas lesiones. Por su lado los avances en la ecografía fetal han permitido que se detecten el $50 \%$ de los casos desde la vida fetal, logrando que a estos niños se les extirpe la lesión durante las primeras semanas de vida y su pronóstico de vida mejore. La presente revisión literaria, busca actualizar conceptos sobre los últimos descubrimientos en la fisiopatología, detección y manejo de la MCVAP.
\end{abstract}

Palabras clave: Malformación adenomatoide quística congénita del pulmón; Anomalias del sistema respiratorio. (Fuente: DeCS BIREME).

\section{CONGENITAL PULMONARY AIRWAY MALFORMATION}

\begin{abstract}
Congenital pulmonary airway malformation (MCVAP), formerly known as congenital cystic adenomatoid pulmonary malformation (MAQP), belongs to the group of congenital pulmonary malformations, representing $95 \%$ of these. It is characterized by varying degrees of respiratory distress according to the level of the airway that compromises the injury and may be incompatible with life until it goes unnoticed until early adulthood. Advances in molecular biology have discovered that there is an imbalance between proliferating and apoptotic genes within these lesions, disrupting the segmentation of the bronchial tree, triggering the formation of these lesions. On the other hand, advances in fetal ultrasound have allowed $50 \%$ of cases to be detected from fetal life, causing these children to have their lesions removed during the first weeks of life and their improved life prognosis. This literary review seeks to update concepts on the latest discoveries in the pathophysiology, detection and management of MCVAP.
\end{abstract}

Key Words: Cystic Adenomatoid malformation of lung congenital; Respiratory system abnormalities. (Source: MeSH NLM).

\section{INTRODUCCIÓN}

malformación adenomatoidea quística pulmonar (MAQP), es una enfermedad rara con una incidencia de 1 por 8300 a 35000 nacidos vivos ${ }^{1}$ y corresponde a la más común de las malformaciones congénitas pulmonares. Fue descrita por primera vez por Ch'in y Tang en 1949. Años después en 1977, Stocker brindo su primera clasificación en 3 tipos de lesión según el tamaño del quiste y su tipo celular: tipo 1 bronquiolar, tipo 2 bronquiolar/alveolar, y tipo 3 células ductales. Bajo esta clasificación más del $65 \%$ eran del tipo 1, mientras que el tipo 2 comprometía $20-25 \%$ y tipo 3 el $8 \% 2$ infection or pneumothorax. Asymptomatic cysts present a therapeutic dilemma: surgical intervention and l"conservativel" observation have advocates. The risk of malignancy in such cysts is considered by some an indication for surgical intervention and is reviewed in this paper. Pleuropulmonary blastoma (PPB. Sin embargo, en el 2002, al encontrar múltiples lesiones no compatibles con dichos parámetros, propuso una nueva clasificación siguiendo una aproximación topográfica planteando 5 tipos de MCVAP (Tabla 1); así mismo sugirió replantear el nombre ya que no todas estas lesiones son adenomatoideas o quísticas como lo indicaba su nombre cambiándolo a MCVAP como lo conocemos hoy. ${ }^{3}$

Etiología Por mucho tiempo se asumió que estas eran lesiones hamartomatosas esporádicas benignas, su formación no se relaciona con factores maternos, raza, edad o exposición. En algunas series las lesiones presentes en la infancia tienen ligera preponderancia masculina. Kumasaki en el 2006 realizo una investigación en la que propuso que las malformaciones congénitas de pulmón tenían u origen en la atresia bronquial ${ }^{4}$ ya que solía encontrar esta lesión asociada a las malformaciones ${ }^{5}$.

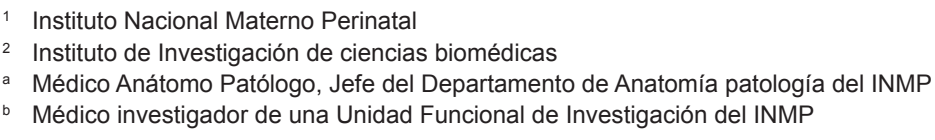

Citar como: Tasayco JC, Saldaña-Díaz C, Roque JC, Paredes JS, Espinola-Sánchez M. Malformación congénita de la vía aérea pulmonar. Rev Peru Investig Matern Perinat 2020; 9(1): 45-8. DOI https://doi.org/10.33421/inmp.2020182 
Los avances de la genética e inmunohistoquimica han permitido plantear 2 rutas fisiopatológicas de MCVAP. La hipótesis ambiental plantea que la expresión persistente de marcadores de desarrollo del pulmón joven causado por potenciales efectos genéticos llevarían a una interrupción focal y temporal de la morfogénesis pulmonar. Por otro lado los hallazgos histológicos han permitido plantear la hipótesis obstructiva, la cual cosiste en una obstrucción focal del árbol bronquial tanto funcional por alguna anormalidad de la peristalsis de las células ciliadas u orgánica por estenosis bronquial; podría generar un aumento de los mediadores y engendrar anormalidades como la MCVAP. Un estudio de MCVAP de fetos y recién nacidos demostró que tenían el doble de proliferación celular y 5 veces más de cuerpos apoptóticos del que tenían los tejidos pulmonares fetales y neonatológicos ${ }^{6}$.

Otro elemento encontrado como target de MCVAP es el factor neurotrófico derivado de células gliales (GDNF), un factor de crecimiento que es ampliamente expresado en órganos con un desarrollo caracterizado por interacción entre epitelio y mesénquima. En un reporte la GDNF se detectó en las células epiteliales de los MCVAP y no se detectó en tejido pulmonar normal de infantes mayores de 4 meses a 3 años de edad ${ }^{7}$. Los genes claves conocidos por tener potencialmente un rol en la morfogénesis temprana del pulmón estarían también involucradas en la patogénesis de la MCVAP ${ }^{8}$. Entre estos se encuentran el gen del factor de transcripción tiroideo (Nkx2), ${ }^{9}$ surrounding normal lung tissue, and both preneoplastic and neoplastic lesionsel gen de la región determinante del sexo 2 (SOX 2), el gen HOX (Hoxb-5), el gen Ying Yang 1 (Yy1), Factor de crecimiento fibroblasto (FGF 1, 9,7) 101112.
Clasificación Los 5 diferentes tipos de MCVAP según Stocker planteada en el 2002 suceden a diferentes niveles del árbol traquebronquial y en diferentes momentos del desarrollo pulmonar ${ }^{13}$. Su compromiso clínico depende de la localización de la lesión, llegando a ser asintomáticos y muchas veces son hallazgos incidentales como en las lesiones tipo 2 y algunas tipo 1 y 2, hasta llegar a ser incompatibles con la vida como la tipo 0 o traer una fuerte carga oncogénica como la tipo 1 y 4 . Las tipo 1 y 2 suelen ser las más frecuentes y comparten como principal síntoma el distress respiratorio en diferentes grados de severidad ${ }^{3}$.

Diagnóstico Un estudio del 2016 demostró que un $85.7 \%$ de las lesiones podía ser detectadas con ecografía a una edad gestacional media de 21 a 24 semanas. Se puede observar como una masa dentro del pulmón. Se ha categorizado prenatalmente como 3 tipos: tipo 1 (25\% de los casos) lesión macroquística y multiquística caracterizada por uno o más quistes de tamaño variable de un máximo de $10 \mathrm{~cm}$, tipo 2 (25\% de los casos) una masa mixta con quistes y un área adyacente con ecogenicidad aumentada de tipo $3(50 \%)$ una lesión microquística de menos de $5 \mathrm{~mm}$, anecogénica dentro del pulmón fetal. La hiperecogenicidad no es específica y no predice el diagnostico histológico. La precisión de la ecografía puede alcanzar 90 y $77 \%$ para sensibilidad y especificidad respectivamente. Su principal diagnóstico diferencial, el secuestro pulmonar, puede identificarse visualizando el origen del vaso nutricio viniendo directo de la aorta con la ecografía Doppler ${ }^{4}$.

Otra herramienta importante en el diagnóstico prenatal es la resonancia magnética, tanto su sensibilidad y

Tabla 1. Clasificación de Malformación congénita de vía aérea pulmonar según Stocker $2002^{3}$

\begin{tabular}{|c|c|c|c|}
\hline Tipo & Macroscopía & Microscopía & Compromiso fetal \\
\hline $\begin{array}{l}\text { Tipo } 0 \\
\text { Traqueo-bronquial: } \\
<2 \% \\
\text { Displasia acinar }\end{array}$ & $\begin{array}{l}\text { Pulmones pequeños y firmes con } \\
\text { superficie granular } \\
\text { Peso pulmón es } 30-50 \% \text { del } \\
\text { peso esperado } \\
\text { Parénquima solido con espacios } \\
\text { vacios de }<0,1 \mathrm{~cm} \text {. }\end{array}$ & $\begin{array}{l}\text { Quistes pequeños } 0.5 \mathrm{~cm} \text { máximo. } \\
\text { Revestido de epitelio } \\
\text { pseudoestratificado ciliado } \\
\text { Células mucosas y cartilaginosas } \\
\text { presentes, musculo esquelético } \\
\text { ausente. }\end{array}$ & Incompatible con la vida. \\
\hline $\begin{array}{l}\text { Tipo } 1 \\
\text { Bronquial- } \\
\text { bronquiolar: } \\
60-70 \% \\
\text { Lesión quística grande }\end{array}$ & $\begin{array}{l}95 \% \text { limitada a un lóbulo. } \\
\text { Tamaño de } 1-10 \mathrm{~cm} \\
\text { Paredes delgadas } \\
\text { Rodeada de membrana lisa. } \\
\text { Multiloculados. }\end{array}$ & $\begin{array}{l}\text { Revestido de epitelio columnar } \\
\text { pseudoestraatificado } \\
\text { Epitelio contraído de apariencia } \\
\text { polipoidea } \\
35-50 \% \text { de los casos hay focos de } \\
\text { células mucinosas. }\end{array}$ & $\begin{array}{l}\text { Desplazamiento de estructuras } \\
\text { adyacentes según tamaño. } \\
\text { Focos mucinosos asociados a } \\
\text { carcinoma broquioalveolar. } \\
\text { Buen pronóstico tras resección. }\end{array}$ \\
\hline $\begin{array}{l}\text { Tipo } 2 \\
\text { Bronquiolar: } \\
\text { 15-20\% Lesiones } \\
\text { quísticas pequeñas }\end{array}$ & $\begin{array}{l}\text { Múltiples quistes de } 0.5 \text { a } 1.5 \\
\mathrm{~cm} \text { de diámetro rodeadas de } \\
\text { membrana lisa que se une al } \\
\text { parénquima normal. }\end{array}$ & $\begin{array}{l}\text { Revestido de epitelio cuboidal y } \\
\text { columnar parcialmente separado } \\
\text { de estructuras tipo alveolo ductales } \\
\text { irregulares. } \\
\text { Células mucinosas ausentes. }\end{array}$ & $\begin{array}{l}60 \% \text { asociado a anomalías congénitas } \\
50 \% \text { asociado a secuestro pulmonar }\end{array}$ \\
\hline $\begin{array}{l}\text { Tipo } 3 \\
\text { Bronquiolar- alveolo } \\
\text { ductal: } \\
5-10 \% \\
\text { Lesión adenomatoidea }\end{array}$ & $\begin{array}{l}\text { Compromete un lóbulo entero o } \\
\text { incluso el pulmón entero. } \\
\text { Masa voluminosa no quística } \\
\text { de } 0,5 \text { a } 1.5 \mathrm{~cm} \text { con paredes } \\
\text { delgadas }\end{array}$ & $\begin{array}{l}\text { Estructuras tipo bronquiolo/alveolar } \\
\text { dispersos al azar revestidas por } \\
\text { epitelio cubidal bajo } \\
\text { Cartílago y células mucinosas } \\
\text { ausentes. }\end{array}$ & $\begin{array}{l}\text { Casi exclusivo de hombres. } \\
80 \% \text { asociado a polihidramnios } \\
\text { maternos. } \\
\text { Óbito fetal, distres respiratorio severo o } \\
\text { muerte neonatal. }\end{array}$ \\
\hline $\begin{array}{l}\text { Tipo } 4 \\
\text { Acinar distal } \\
5-10 \%\end{array}$ & $\begin{array}{l}\text { Quistes de paredes delgadas } \\
\text { Periféricos, distendidos y } \\
\text { semitransparentes }\end{array}$ & $\begin{array}{l}\text { Revestidos de células epiteliales } \\
\text { planas tipo alveolares tipo } 1 \text { y tipo } 2 . \\
\text { Células mucinosas y musculares } \\
\text { ausentes. }\end{array}$ & $\begin{array}{l}\text { Leve distress respiratorio, infecciones } \\
\text { recurrentes o neumotórax a tensión, } \\
\text { incidental } \\
\text { Asociado a blastoma pleuropulmonar. }\end{array}$ \\
\hline
\end{tabular}


especificidad pueden alcanzar hasta un 95\% de precisión. Sin embargo no es una médica costo efectiva ${ }^{15}$.

El crecimiento de la MCVAP suele detenerse alrededor de las 28 semanas, 11 a $49 \%$ de estas puede desaparecer espontáneamente, $18-42 \%$ encogerse y 33 a $44 \%$ progresar. Se ha observado que $44.7 \%$ de los casos diagnosticados prenatalmente fueron confirmados postnatalemente. Sin embargo es importante detectar complicaciones prenatalmente como hidrops, shift mediastinal, hidramnios o muerte fetal ${ }^{16}$.

Crombleholme et al. Desarrollo un índice llamado el índice de volumen de la MCVAP (CVR por sus siglas en ingles). El cual estandarizada el volumen de una MCVAP según la edad gestacional. Representaba el índice entre el volumen estimado de la MCVAP y la circunferencia cefálica, siendo el volumen determinado de la estimación de la MCVAP como una elipse y multiplicando la longitud por la altura por el ancho por 0.52. Múltiples autores observaron que un CVR mayor de 1.6 era altamente predictivo de hidrops ${ }^{14}$.

La indicación de cirugía fetal depende los hallazgos ecocardiográficos. Una regurgitación significativa valvular, disminución de la función ventricular o cambios en el doppler parecen predecir un resultado adverso. El tratamiento esteroideo ha sido usado en lesiones microquísticas con hidrops con menor riesgo de prematuridad, de requerimiento de ventilador y un mejor resultado ${ }^{15}$.

El diagnóstico postnatal es principalmente hecho en pacientes con distress respiratorio en distinto grado de severidad que no mejora o empeora al nacer o con neumonías a repetición, sibilantes durante los primeros meses de vida. Incluso algunos casos suelen manifestarse durante la niñez o la adultez. En la radiografía la MCVAP se puede ver como una masa radio opaca, puede detectar hasta un 50-60\% de MCVAP incluso de manera incidental. Por otro lado la TEM con contraste puede detectar el $100 \%$ de las MCVAP ${ }^{17}$.

Progresión a malignidad El pleuroblastoma pulmonar (PBP) es un tumor raro, cuya incidencia es difícil de calcular debido a sus escasos casos. Se divide en tres tipos, tipo I quístico, tipo II, quístico y sólido y tipo III sólido. Hasta hace poco no existía ningún criterio clínico o radiológico o biológico para distinguir entre PBP tipo I y MCVAP tipo 4 aludiendo una relación de posible transformación maligna de la MCVAP. No existe límite de edad para la evolución a PBP y es impredecible ${ }^{18}$.

Por otro lado se ha observado que las células mucinosas de los focos mucinosos encontrados en MCVAP tipo 1 y tipo 2 comparten el mismo perfil inmunológico que los adenocarcinomas bronquioalveolares, expresando MUC5AC, CK7, MUC1, CDX2, BRAF y ALK; asi como también mutaciones en el gen KRAS y LOH con o sin alteraciones de los microsaletelites del locus p16 ink 4, todo esto justificaría su consideración como lesiones precursoras de adenocarcinoma ${ }^{19-20}$.

Conclusiones. En los últimos años se han hecho múltiples avances radiológicos como patológicos para comprender mejor las MCVAP. Es importante mantenerse actualizado dado que corresponde a la malformación congénita pulmonar más frecuente, principalmente por su diagnóstico prenatal y su potencial maligno. El debate permanece abierto sobre el manejo adecuado de esta malformación dado que un buen porcentaje se mantiene asintomático, por lo que su resección suele ser discutida. Sin embargo los últimos hallazgos inmunohistoquimicos están probando un claro perfil premaligno de estas lesiones por lo que la tendencia actual es a reseccionarlos. Es necesario continuar haciendo revisiones de los últimos hallazgos moleculares para poder llegar a conclusiones más certeras con respecto a este tema, así mismo corroborar en nuestra población le incidencia de esta enfermedad para manejar información local.

Financiamiento: Autofinanciado.

Conflicto de interés: Los autores declaran no tener algún conflicto de intereses.

\section{REFERENCIAS BIBLIOGRÁFICAS}

1. Leblanc C, Baron M, Desselas E, Phan MH, RybakA, Thouvenin $G$, et al. Congenital pulmonary airway malformations: state-ofthe-art review for pediatrician's use. Eur J Pediatr. diciembre de 2017;176(12):1559-71.

2. Priest JR, Williams GM, Hill DA, Dehner LP, Jaffe A. Pulmonary cysts in early childhood and the risk of malignancy. Pediatric Pneumology [Internet]. enero de 2009 [citado 20 de diciembre de 2019];44(1):14-30. Disponible en: https://www. ncbi.nlm.nih.gov/pubmed/19061226

3. JT Stocker. Congenital pulmonary airway malformation-a new name for and an expanded classification of congenital cystic adenomatoid malformation of the lung. En Department of pathology National Defense Medical College, Tokorozawa, Saitama, Japon: Histopathology; p. 424-58. (Suplemento 2; vol. 41).

4. Kunisaki SM, Fauza DO, Nemes LP, Barnewolt CE, Estroff JA, Kozakewich HP, et al. Bronchial atresia: the hidden pathology within a spectrum of prenatally diagnosed lung masses. J Pediatr Surg. enero de 2006;41(1):61-5; discussion 61-65.

5. Aditi Jain, KAnand, Saurabh Singla, Ashok Kumar. Congenital Cystic Lung Diseases. J Clin Imaging Sci [Internet]. 2013 [citado 20 de diciembre de 2019];3(5). Disponible en: https:// www.ncbi.nlm.nih.gov/pmc/articles/PMC3625881/

6. Leblanc $C$, Baron $M$, Desselas $E$, Phan $M H$, Rybak A, Thouvenin G, et al. Congenital pulmonary airway malformations: state-of-the-art review for pediatrician's use. Eur J Pediatr. diciembre de 2017;176(12):1559-71.

7. Barazzone-Argiroffo C, Lascano Maillard J, Vidal I, Bochaton-Piallat ML, Blaskovic S, Donati $\mathrm{Y}$, et al. New insights on congenital pulmonary airways malformations revealed by proteomic analyses. Orphanet J Rare Dis. 28 de 2019;14(1):272.

8. Zhu H, Liu D, Jia H. Analysis of Wnt7B and BMP4 expression patterns in congenital pulmonary airway malformation. Pediatr Pulmonol. marzo de 2020;55(3):765-70. 
9. Stacher E, Ullmann R, Halbwedl I, Gogg-Kammerer M, Boccon-Gibod L, Nicholson AG, et al. Atypical goblet cell hyperplasia in congenital cystic adenomatoid malformation as a possible preneoplasia for pulmonary adenocarcinoma in childhood: A genetic analysis. Hum Pathol. mayo de 2004;35(5):565-70.

10. Qu Y, Liu D, Jia H, Zhou X. Expression Analysis of ACSL5 and Wnt2B in Human Congenital Pulmonary Airway Malformations. J Surg Res. 2018;232:128-36.

11. Swarr DT, Peranteau WH, Pogoriler J, Frank DB, Adzick NS, Hedrick HL, et al. Novel Molecular and Phenotypic Insights into Congenital Lung Malformations. Am J Respir Crit Care Med [Internet]. 15 de mayo de 2018 [citado 13 de febrero de 2020];197(10):1328-39. Disponible en: https://www.ncbi.nlm. nih.gov/pmc/articles/PMC5955056/

12. Nasr A, Himidan S, Pastor AC, Taylor G, Kim PCW. Is congenital cystic adenomatoid malformation a premalignant lesion for pleuropulmonary blastoma? J Pediatr Surg. junio de 2010;45(6):1086-9.

13. Teresa Berrocal, Carmen Madrid Susana Novo, Julia Gutierrez, Antonia Arjonilla, Nieves Gomez-Leon. Congenital Anomalies of the Tracheobronchial Tree, Lung, and Mediastinum: Embryology, Radiology, and Pathology | RadioGraphics. RadioGraphics [Internet]. febrero de 2003 [citado 20 de diciembre de 2019];24(1):e17. Disponible en: https://pubs.rsna.org/doi/10.1148/rg.e17?url_ver=Z39.882003\&rfr_id=ori:rid:crossref.org\&rfr_dat=cr_pub\%3dpubmed

14. Thakkar HS, Durell J, Chakraborty S, Tingle B-L, Choi A, Fowler DJ, et al. Antenatally Detected Congenital Pulmonary Airway Malformations: The Oxford Experience. Eur J Pediatr Surg. agosto de 2017;27(4):324-9.

15. Shanmugam G, MacArthur K, Pollock JC. Congenital lung malformations--antenatal and postnatal evaluation and management. Eur $\mathrm{J}$ Cardiothorac Surg. enero de 2005;27(1):45-52.
16. Krous HF, Harper PE, Perlman M. Congenital cystic adenomatoid malformation in bilateral renal agenesis. Its mitigation of Potter's syndrome. Arch Pathol Lab Med. julio de 1980;104(7):368-70.

17. Ortac R, Diniz G, Yildirim HT, Aktas S, Karaca I. Retrospective Evaluation of Children with Congenital Pulmonary Airway Malformation: A Single Center Experience of 20 Years. Fetal Pediatr Pathol. 2016;35(3):143-8.

18. MacSweeney F, Papagiannopoulos K, Goldstraw P, Sheppard MN, Corrin B, Nicholson AG. An assessment of the expanded classification of congenital cystic adenomatoid malformations and their relationship to malignant transformation. Am J Surg Pathol. agosto de 2003;27(8):1139-46.

19. Cangiarella J, Greco MA, Askin F, Perlman E, Goswami S, Jagirdar J. Congenital cystic adenomatoid malformation of the lung: insights into the pathogenesis utilizing quantitative analysis of vascular marker CD34 (QBEND-109 and cell proliferation marker MIB-1. Mod Pathol [Internet]. 1995 [citado 20 de diciembre de 2019];8(9):913-8. Disponible en: https://www.ncbi.nlm.nih.gov/pubmed/8751331

20. Stephanov O, Robert Y, De Fraipont F, Piolat C, Sartelet $\mathrm{H}$. Mucinous adenocarcinoma with lepidic pattern and with K-RAS mutation in a newborn with antenatal diagnosis of congenital pulmonary airway malformation. Histopathology. 2018;72(3):530-1.

Correspondencia:

Juan Carlos Tasayco

Dirección: Jr. Santa Rosa 941 Lima

Correo: jcts80@hotmail.com

Teléfono: 941979660 